\title{
What does a neuron "see"? Limitations imposed by the statistics of afferent inputs to a neuron Mark D Humphries
}

Address: Adaptive Behaviour Research Group, University of Sheffield, Sheffield, UK

Email: Mark D Humphries - m.d.humphries@shef.ac.uk

from Eighteenth Annual Computational Neuroscience Meeting: CNS*2009

Berlin, Germany. 18-23 July 2009

Published: I 3 July 2009

BMC Neuroscience 2009, I0(Suppl I):O8 doi:10.1 I86/I47I-2202-10-SI-O8

This abstract is available from: http://www.biomedcentral.com/I47I-2202/I0/SI/O8

(C) 2009 Humphries; licensee BioMed Central Ltd.

Neurons encode information in their spike trains. In a range of neural systems, every spike train and potentially each spike is supremely important [1], so important that, given appropriate input, many neurons can reliably repeat their spike timing with startling accuracy [2]. Yet a target neuron sees many such spike trains in its afferent input. And the statistics of such a group of spike-trains are well described by simple models that pay no heed to the single spike or even the single spike train. How then do we reconcile the success of these simple models with the complexity of encoding within and decoding of spike trains $[1,3]$ ?

First, we set out to understand just how accurate these simple models are. They are often used for qualitative understanding of problems [1] and that is certainly an approach used here. But we show that these simple models can be pushed hard before they break down and thereby can give quantitative insight. We build a framework that directly generates the set of spike-events that occur across all the target neuron's inputs and that encapsulate many simple models of the individual spike trains. Nonetheless, these spike-event generators are sufficiently simple for us to gain analytical insight into exactly how the structure of the input to a neuron is dependent on the number, rate and correlation of the individual spike trains. As an example, we use this to model the global properties that arise from weak pair-wise correlations between spike trains [4].
The framework's most interesting prediction is that the global statistics of the afferent input to a single neuron converge for a wide range of properties of the individual afferent spike trains, thus obscuring potentially important properties of those spike trains. How then does information carried by individual spike trains become decoded into the output of a neuron? This questions stands in contrast to much previous work that has focused on decoding the spike train correlates of continuous stimuli $[1,3]$. We show, because of the obscuring effects on individual trains, our framework predicts that strongly asymmetric distributions of synaptic conductances are necessary for reliable reproduction of output spike trains in response to common components of different sets of inputs [2]. Thus, we posit this as the reason for such distributions in the vertebrate brain, recently described in many neural circuits [5].

\section{Acknowledgements}

This work was funded by the EU Framework 6 Project IST-0278I9-IP.

\section{References}

I. Rieke F, Warland D, de Ruyter van Stevninck R, Bialek W: Spikes: Exploring the neural code Cambridge, MA: MIT Press; 1997.

2. Ermentrout GB, Galan RF, Urban NN: Reliability, synchrony and noise. Trends Neurosci 2008, 3 I:428-434.

3. Eliasmith C, Anderson CH: Neural Engineering: Computation, Representation, and Dynamics in Neurobiological Systems Cambridge, MA: MIT Press; 2003.

4. Schneidman E, Berry MJ, Segev R, Bialek W: Weak pairwise correlations imply strongly correlated network states in a neural population. Nature 2006, 440: $1007-1012$.

5. Barbour B, Brunel N, Hakim V, Nadal J-P: What can we learn from synaptic weight distributions? Trends Neurosci 2007, 30:622-629. 